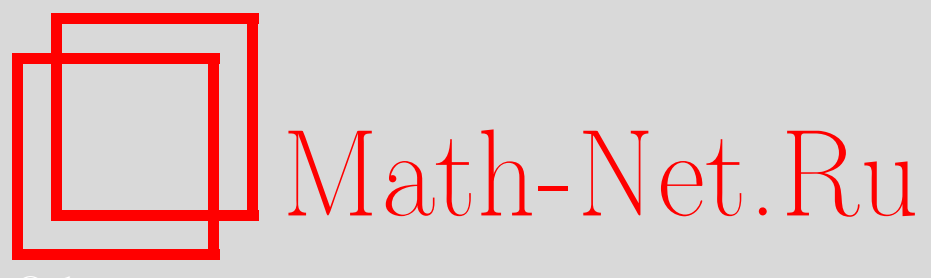

С. В. Михайлов, Тип трансцендентности для почти всех точек $m$-мерного комплексного пространства, $У М H$, 2008, том 63, выпуск 2, 175-176

DOI: https://doi.org/10.4213/rm9062

Использование Общероссийского математического портала Math-Net.Ru подразумевает, что вы прочитали и согласны с пользовательским соглашением http://www . mathnet.ru/rus/agreement

Параметры загрузки:

IP: 54.237 .206 .68

26 апреля 2023 г., 13:44:51

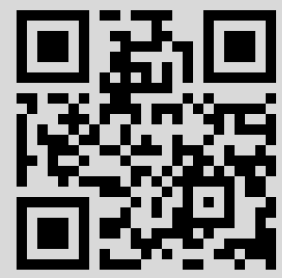




\section{Тип трансцендентности для почти всех точек $m$-мерного комплексного пространства}

\section{С. В. Михайлов}

Пусть $P$ - многочлен с целыми коэффициентами, зависящий от $m$ переменных. Обозначим $\operatorname{deg} P$ степень $P$ по совокупности переменных, $H(P)$ - максимум модулей коэффициентов $P$ и $t(P)=\operatorname{deg} P+\ln H(P)$ - тип многочлена $P$. Пусть $\bar{\xi} \in \mathbb{C}^{m}$ - точка с алгебраически независимыми (над $\mathbb{Q}$ ) координатами, $\tau>0$ - вещественное. Будем говорить, что $\bar{\xi}$ имеет тип трансцендентности $\leqslant \tau$, если для любого многочлена $P \in \mathbb{Z}\left[x_{1}, \ldots, x_{m}\right], P \not \equiv 0$, выполнено неравенство

$$
|P(\bar{\xi})|>e^{-c t(P)^{\tau}},
$$

где $c>0$ - константа, зависящая, вообще говоря, от $\bar{\xi}$, но не от многочлена $P$.

Это определение (для $m=1$ ) было дано С. Ленгом в 1966 г. (см. [1; гл. 5, §1]).

В 1971 г. К. Малер [2] выдвинул гипотезу о том, что почти все вещественные числа имеют тип трансцендентности $\leqslant 2$. Это предположение было доказано Ю. В. Нестеренко [3] в 1974 г. В этой же работе доказывается, что почти все точки $\bar{\xi} \in \mathbb{R}^{m}$ имеют тип трансцендентности $\leqslant m+2$, и выдвигается предположение, что на самом деле почти все точки $\bar{\xi} \in \mathbb{R}^{m}$ имеют тип трансцендентности $\leqslant m+1$.

Аналогичная гипотеза (но уже в комплексном случае) была высказана Г. В. Чудновским в 80-х годах прошлого века (см. [4; гипотеза 1.3]) и доказана Ф. Аморозо [5] в 1990 г. Доказательство, изложенное в [5], существенно использует "комплексность" ситуации, и его не удается адаптировать к вещественному случаю. В работе [6] автором было получено доказательство того, что почти все точки $\bar{\xi} \in \mathbb{R}^{m}$ имеют mun трансцендентности $\leqslant m+1$, причем это было сделано методом, который может быть использован и в комплексном случае. Цель настоящей заметки - дать новое доказательство гипотезы Чудновского, используя технику работы [6]. Итак, имеет место

Теорема 1. Почти все точки $\bar{\xi} \in \mathbb{C}^{m}$ (в смысле $2 m$-мерной меры Лебега) имеют тип трансцендентности $\leqslant m+1$.

Используя принцип Дирихле, можно показать, что точка $\bar{\xi} \in \mathbb{C}^{m}$ не может иметь тип трансцендентности $\leqslant m+1-\varepsilon$, т. е. оценка теоремы 1 точна.

Схема доказАтельствА. Требуется доказать, что $2 m$-мерная лебегова мера $\mu$ множества $\Omega$ точек $\bar{\xi} \in \mathbb{C}^{m}$, которые не удовлетворяют условию (1) с $\tau=m+1$, равна нулю. Обозначим через $\Omega_{0}$ пересечение множества $\Omega$ с единичным кубом $B=\left\{\bar{\xi} \in \mathbb{C}^{m}:|\bar{\xi}| \leqslant 1\right\}$. Можно показать, что равенство $\mu\left(\Omega_{0}\right)=0$ влечет за собой равенство $\mu(\Omega)=0$ (достаточно заметить, что при умножении точки $\bar{\xi} \in \mathbb{C}^{m}$ на натуральное число $n$ ее тип трансцендентности не меняется). Поэтому достаточно доказать равенство $\mu\left(\Omega_{0}\right)=0$. Для этого нам потребуются вспомогательные множества. Обозначим через $S_{0}$ множество точек $\bar{\xi} \in B$, координаты которых алгебраически зависимы. Пусть $\tau_{1}, \tau_{2}$ - положительные вещественные числа, $n$ - натуральное число. Обозначим через $B_{n}\left(\tau_{1}, \tau_{2}\right)$ множество точек $\bar{\xi} \in B$, для каждой из которых существует многочлен $Q \in \mathbb{Z}\left[x_{1}, \ldots, x_{m}\right]$ такой, что

$$
t(Q) \leqslant n, \quad|Q(\bar{\xi})| \leqslant e^{-\tau_{1} n^{m+1}}, \quad \max _{1 \leqslant i \leqslant m}\left|\frac{\partial Q}{\partial x_{i}}(\bar{\xi})\right| \geqslant e^{-\tau_{2} n^{m+1}} .
$$

И пусть $S\left(\tau_{1}, \tau_{2}\right)$ - множество точек, каждая из которых содержится в бесконечном числе множеств $B_{n}$. Можно доказать (см. [6; лемма 3]), что при $\tau_{1}>\tau_{2}+1 / m$ ! мера множества $S\left(\tau_{1}, \tau_{2}\right)$ равна нулю. 
Далее, пусть $\mathbb{Q}[X]$ - кольцо многочленов от переменных $x_{0}, \ldots, x_{m}$ над $\mathbb{Q}$. Для любого однородного несмешанного идеала $I$ кольца $\mathbb{Q}[X]$ можно определить понятия степени $\operatorname{deg} I$ идеала, логарифмической высоты $h(I)$ идеала и величины идеала в точке $\bar{\xi}^{\prime}$ проективного комплексного пространства $\mathbb{C}^{m+1}$, обозначаемой $\left|I\left(\bar{\xi}^{\prime}\right)\right|$. Подробные определения можно найти в работе [7]. Эти величины по своим свойствам напоминают аналогичные характеристики многочлена. По аналогии с типом многочлена можно определить понятие тuпа идеала $I$ формулой $t(I)=\operatorname{deg} I+h(I)$.

ЛЕмма 1. Пусть $\mathfrak{p} \subset \mathbb{Q}[X]-$ простой однородный идеал, $r=\operatorname{dim} \mathfrak{p}+1 \geqslant 1$. Пусть $L=\min _{E \in \mathfrak{p}} t(E)$, где минимум берется по всем однородным многочленам идеала $\mathfrak{p}$. Тогда существует однородный многочлен $F \in \mathfrak{p} \cap \mathbb{Z}\left[x_{0}, \ldots, x_{m}\right]$ такой, что некоторая его частная производная не лежит в идеале р и при этом $t(F) \leqslant c_{1} L, c_{1}=c_{1}(m)$.

ДоказАТеЛЬСтво. См. лемму 6 в [6].

Пусть $\lambda=\lambda(m)>0$ - достаточно большое вещественное число. Обозначим $A_{r}$ множество точек $\bar{\xi} \in B \backslash S_{0}$, для которых существует бесконечная последовательность различных однородных несмешанных идеалов $I \subset \mathbb{Q}[X]$ таких, что

$$
\operatorname{dim} I=r-1, \quad \ln \left|I\left(1, \xi_{1}, \ldots, \xi_{m}\right)\right| \leqslant-\lambda^{3^{2 r}}(t(I))^{\frac{m+1}{m+1-r}}, \quad 1 \leqslant r \leqslant m .
$$

Положим по определению $A_{0}=\varnothing$. Далее, обозначим через $B_{r}$ множество точек $\bar{\xi} \in B \backslash S_{0}$, для которых существует бесконечная последовательность различных однородных простых идеалов $\mathfrak{p} \subset \mathbb{Q}[X]$ таких, что

$$
\operatorname{dim} \mathfrak{p}=r-1, \quad \ln \left|\mathfrak{p}\left(1, \xi_{1}, \ldots, \xi_{m}\right)\right| \leqslant-\lambda^{3^{2 r-1}}(t(\mathfrak{p}))^{\frac{m+1}{m+1-r}}, \quad 1 \leqslant r \leqslant m .
$$

И, наконец, положим $S=S\left(\tau_{1}, \tau_{2}\right), \tau_{1}=(1 / 4) \lambda\left(4 c_{1}\right)^{-m-1}, \tau_{2}=\tau_{1}-2 / m !$, где $c_{1}-$ константа из леммы 1 . Отметим, что $\mu(S)=0$, поскольку $\tau_{1}>\tau_{2}+1 / m$ ! .

Лемма 2. Справедливы следующие включения:

$$
A_{r} \subset B_{r}, \quad B_{r} \subset A_{r-1} \cup S, \quad r=1, \ldots, m ; \quad \Omega_{0} \subset A_{m} \cup S_{0} .
$$

ДокАЗАтельство. См. леммы 7, 8 и раздел “доказательство теоремы 1" в [6].

Из леммы 2 следует, что справедлива цепочка включений

$$
\Omega_{0} \backslash S_{0} \subset A_{m} \subset B_{m} \subset A_{m-1} \cup S \subset \cdots \subset A_{1} \cup S \subset B_{1} \cup S \subset S .
$$

Следовательно, $\Omega_{0} \subset S \cup S_{0}$ и $\mu\left(\Omega_{0}\right)=0$, что доказывает теорему 1.

\section{Список литературы}

[1] S. Lang, Introduction to transcendental numbers, Addison-Wesley, Reading-London-Don Mills, 1966. [2] K. Mahler, Acta Arith., 18 (1971), 63-76. [3] Ю. В. Нестеренко, Матем. заметки, 15:3 (1974), 405-414; англ. пер.: Yu. V. Nesterenko, Math. Notes, 15 (1974), 234-240. [4] G.V. Chudnovsky, Contribution to the theory of transcendental numbers, Math. Surveys Monogr., 19, Amer. Math. Soc., Providence, RI, 1984. [5] F. Amoroso, Acta Arith., 56:4 (1990), 345-364. [6] С. В. Михайлов, Матем. сб., 198:10 (2007), 67-88; англ. пер.: S. V. Mikhailov, Sb. Math., 198:10 (2007), 1443-1463. [7] Ю. В. Нестеренко, Аналитическая теория чисел и приложения, Тр. МИАН, 218, Наука, М., 1997, 299-334; англ. пер.: Yu. V. Nesterenko, Proc. Steklov Inst. Math., 218 (1997), 294-331.

\section{C. В. Михайлов (S. V. Mikhailov)}

Московский государственный университет им. М. В. Ломоносова

E-mail: serjikk@gmail.com
Представлено А. В. Михалёвым Принято редколлегией 29.11.2007 